\title{
LAGRANGIAN CORRELATION AND SPECTRA IN 2D TURBULENCE*
}

\author{
HUA XIA \\ Research School of Physics and Engineering, The Australian National University, Canberra, ACT 0200, \\ Australia \\ hua.xia@anu.edu.au
}

\begin{abstract}
Transition from chaotic flow to turbulent flow is investigated in both the Eulerian and Lagrangian frame of reference. Eulerian spectra of the kinetic energy of the flow show the development of a hierarchy of scales, generation of a broad $k^{-5 / 3}$ spectral range in developed turbulence. In the Lagrangian frame, single particle displacement is dominant by multiple scales for the chaotic flow. In fully developed turbulence, one single scale determines the transport properties, which is related to the most persistent scale in the system, the forcing scale.
\end{abstract}

Keywords: Turbulent flow; transition to turbulence; turbulent diffusion; Lagrangian statistics.

\section{Introduction}

Turbulence is often viewed as a process of energy transfer through a hierarchy of scales, until the energy is dissipated. The existence of the multiple scales in the inertial range is a key component of a turbulent field. How those scales affect particle transport is not well known since the Lagrangian statistics of turbulence is not well investigated. This is due to the difficulties in both Lagrangian measurements and in laboratory turbulence modeling.

It is only very recently laboratory studies of Lagrangian statistics has become viable due to the availability in computational power and high speed high resolution imaging ${ }^{1,2}$ and well characterized laboratory experiments. The discovery of a new turbulence generation mechanism in Faraday wave experiments ${ }^{3,4}$ provides a platform for turbulence generation. It is found that in the Faraday wave driven turbulence (FWT), horizontal motion of the particles moving on the surface of the water shows the two-dimensional turbulence characteristics, that is, well defined energy injection, the inverse energy cascade and the forward enstrophy cascade range. It also supports the generation of the spectral condensation when the system size is small than the dissipation scales. The forcing wave number and strength of the FWT experiments can be varied in a wide range allowing a systematic investigation of parameter dependence of the turbulence properties.

\footnotetext{
* This is an Open Access article published by World Scientific Publishing Company. It is distributed under the terms of the Creative Commons Attribution 3.0 (CC-BY) License. Further distribution of this work is permitted, provided the original work is properly cited.
} 
The FWT experiments are complimented by the electromagnetically driven turbulence (EMT) in fluid layers ${ }^{5}$ which is capable of generating both chaotic and turbulent flows.

The second moment of the single particle dispersion, mean-square dispersion (MSD) of a particle from its initial position $\left\langle\delta r^{2}\right\rangle=\left\langle|\vec{r}(t)-\vec{r}(0)|^{2}\right\rangle$, has been investigated in a wide range of experimental conditions in both FWT and EMT turbulence ${ }^{6}$. It is found that the MSD is diffusive $\left\langle\delta r^{2}\right\rangle=\left\langle|\vec{r}(t)-\vec{r}(0)|^{2}\right\rangle \sim t$ for $t>t_{L}$ for a wide range of experimental parameters, for both the chaotic and turbulent flows. This is the first extensive laboratory studies of the celebrated Taylor's diffusion equation, being only noted before in Refs. 3 and 7. A Lagrangian scale related to the forcing scale has been identified to determine the particle dispersion ${ }^{6}$. More than 30 experiments with varying r.m.s. velocity show the ratio of this Lagrangian scale to the forcing scale is in the range of $0.4-1$, and stabilized at 0.7 in the more turbulent flows (higher r.m.s. velocity).

It is important to reconcile the multi-scale nature of turbulence and the single Lagrangian scale observed for the MSD. In this work, we investigate the transition from chaotic to turbulent flows from the Lagrangian perspective. It is found that the transition from chaos to turbulence is manifested as a transition from multiple Lagrangian scales for chaotic flow to a single Lagrangian scale for turbulence flows, in contrast to that in the Eulerian frame of reference, where the transition to turbulence sees the development of a hierarchy of scales.

\section{Flow Generation}

Two complimentary methods have been used in the experiments to generate flows in a wide range of conditions, from chaotic to turbulent flows.

The first one is the electromagnetically driven turbulence (EMT) in fluid layers, which has been widely used in laboratory modeling of two-dimensional turbulence ${ }^{5}$. The experiments are conducted in the thin layer of electrolyte in the fluid cell. An array of permanent magnets arranged in a check-board fashion is placed underneath the fluid cell. A current driven through the electrolyte interacts with the magnetic field through the Lorenz force $(\mathrm{J} \times \mathrm{B})$, which generates an array of interacting vortices. Those vortices then interact with each other and generate turbulence. The separation between the magnets determines the injection wave number. The strength of the interaction is adjusted by the current density. To reduce the bottom drag and to avoid the influence of the bottom boundary layer, the electrolyte is placed on top of heavier $(\mathrm{SG}=1.8)$ non-conducting fluid (FC-3283 by 3M) immiscible with water. It has been shown that turbulence in the fluid layers is two-dimensional for both the double layer and single layer configuration, even in relatively thick layers ${ }^{8,9}$.

The Faraday wave drive turbulence (FWT) is a newly found way of turbulent generation $^{3,4}$. In this experiment, the horizontal motion of fluid particles is forced by the parametrically excited Faraday waves on the surface of a vertically vibrated liquid (water). Although the motion of floaters in the Faraday waves is three dimensional, its 
horizontal velocity fluctuations show unexpected similarity with two-dimensional turbulence. The inverse cascade is detected by measuring frequency spectra of the Lagrangian velocity, and it is confirmed by computing the third moment of the horizontal velocity fluctuations. The forcing scale of the FWT turbulence is close to the Faraday wavelength determined by the shaker frequency. The main advantage of generating turbulence using Faraday surface waves is the ability to change the forcing scale by simply changing the frequency of the vertical vibration. This method also allows a broader range of horizontal kinetic energies to be achieved4.

In both experiments, tracer particles are placed on the surface of the fluid to visualize fluid motion on the water surface and their positions are recorded using an Andor NEO sCMOS camera at high frequency and spatial resolution. $50 \mu \mathrm{m}$ diameter white Polyamid particles $(\mathrm{SG}=1.03)$ are used for the EMT experiments and black carbon glass particles with a diameter within a range of $150-300 \mu \mathrm{m}$ are used in the FWT experiments. The particles are plasma treated to reduce their intrinsic hydrophobicity. The use of surfactant and plasma treatment makes the particle wettability almost neutral which prevents their clustering and insures homogeneous spreading on the fluid surface. Both the particle image velocimetry (PIV) and particle tracking velocimetry (PTV) techniques have been implement to characterize the flow in both the Eulerian and Lagrangian frame of reference.

To investigate the transition to turbulence, a parameter is needed to characterize the degree of turbulence, similar to the Reynolds number in 3D turbulence. There is no universally accepted definition of the equivalent of Reynolds number in the inverse cascade in $2 \mathrm{D}$ turbulence. A reasonable definition is based on the ratio between two spatial scales or two times ${ }^{10}, e . g$.,

$$
R_{\alpha}=\left(\frac{L_{\alpha}}{L_{f}}\right)^{2 / 3} \sim \frac{\eta_{I}^{1 / 3}}{\alpha}
$$

where $L_{\mathrm{f}}$ is the small scale forcing scale, $L_{\alpha} \sim \varepsilon^{1 / 2} \alpha^{-3 / 2}$ is the large friction scale, $\alpha$ the friction coefficient and $\eta_{I}$ the enstrophy input. $\varepsilon$ is the energy flux in the inertial range.

The friction coefficient can be experimentally estimated through the decay of the turbulence kinetic energy of the flow5. The energy flux can be derived from the measurement of third-order moment of the velocity difference ${ }^{11}$ through the Kolmogorov flux relation $S_{3}=\varepsilon r$. In the experiments described in this work, the measured $R_{\alpha}$ varies from $\sim 1$ to 10 .

\section{Eulerian and Lagrangian Spectra}

The wave number spectra of the flow have been measured using the PIV technique. In Fig. 1, the wave number spectra of the EMT turbulence at several current densities are shown. The derived $R_{\alpha}$ is shown on the graph, which corresponds to current density 

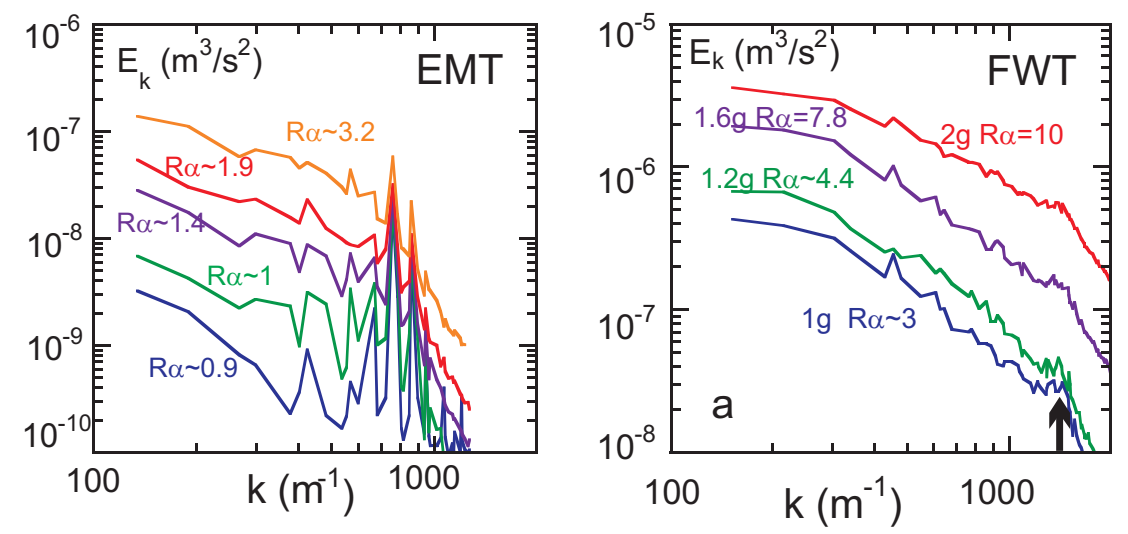

Fig. 1. (a) Wave number spectra of EMT turbulence at several current densities. The current densities are $\mathbf{J}=(0.1,0.2,0.4,0.6,1.2) \times 10^{3} \mathrm{~A} \mathrm{~m}^{-2}$ from bottom to top respectively.(b) Wave number spectra of FWT turbulence at several vertical accelerations.

$\mathbf{J}=(0.1,0.2,0.4,0.6,1.2) \times 10^{3} \mathrm{~A} \mathrm{~m}^{-2}$ from bottom to top respectively. As have been shown before9, at low current density $\left(0.1 \times 10^{3} \mathrm{~A} \mathrm{~m}^{-2}\right)$, the Reynolds number is low, the spectrum is dominant by a peak at the forcing scale. The particle trajectories clearly show the array of vortices generated by the magnetic array, in Fig. 1(a). With increasing current density, the Reynolds number increases, more and more energy are transferred to lower wave number through the inverse energy cascade, until eventually the spectrum at $1.2 \times 10^{3} \mathrm{~A} \mathrm{~m}^{-2}$ shows a $k^{-5 / 3}$ Kraichnan-Kolmogorov scaling.

The FWT experiments have been conducted at $f_{0}=60 \mathrm{~Hz}$ at vertical acceleration from $1 \mathrm{~g}$ to $2 \mathrm{~g}$. The threshold of the parametric excitation is $a_{\mathrm{c}}=0.5 \mathrm{~g}$, which gives the supercriticality parameter of the experiments from 1 to 3 . The Reynolds number as defined in Eq. 1 is from 3 to 10. The flow generated is turbulent from the Eulerian frame, as can be seen from the spectra in Fig. 1(b), which show the $k^{-5 / 3}$ inverse energy cascade range.

During the transition from chaotic to turbulent flow in Eulerian spectral domain, the chaotic flow is dominant by the energy injection scale while the turbulent flow is characterized by a hierarchy of scales in the inertial range due to the inverse energy cascade. The transition to turbulent flow happened at around $R_{\alpha} \sim 2-3$.

In the Lagrangian frame of reference, a spatial Lagrangian velocity autocorrelation function6 has been constructed similarly to the temporal autocorrelation function, $\rho(L)=\left\langle u\left[\vec{r}\left(r_{0}+L\right)\right] u\left[\vec{r}\left(r_{0}\right)\right]\right\rangle / \sigma^{2}$. Moving along a trajectory, a particle's velocity $u(t)$ and its displacement $\delta r(t)$ from the initial position $\mathrm{r}(0)$ are recorded simultaneously. By excluding time we first interpolate measured velocity increments $u[\vec{r}(r)]$ onto a regular array $u(r)$, and then compute the spatial autocorrelation function $\rho(L)$.

We compute a Lagrangian wave number spectrum, which is a Fourier transform of the spatial velocity autocorrelation function, shown in Fig. 2(a). If the Lagrangian 

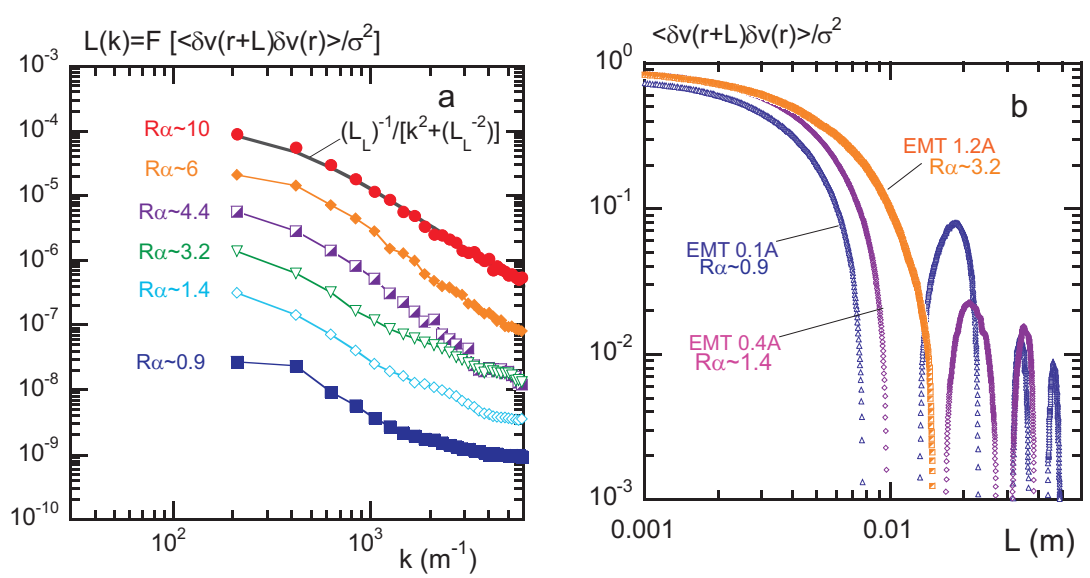

Fig. 2. (a) Lagrangian spectra of the EM turbulence and FW turbulence showing a transition from chaotic to turbulent flow. (b) velocity autocorrelation function.

spectrum can be fitted as a Lorentzian function $L(k) \sim L_{L}^{-1} /\left(k^{2}+L_{L}^{-2}\right)$, the spectrum can be fully characterized by a single scale $L_{\mathrm{L}}$. At higher levels of forcing the Lagrangian wave number spectra are indeed Lorentzian, while this is not the case at low forcing.

Fig. 2(b) shows spatial autocorrelation functions $\rho(L)$ for several cases in Fig. 2(a) in the log-log scale. Though negative values of the oscillating $\$$ rho $\$$ are not seen, this plot reveals the presence of the persistent scales at low Reynolds number $R_{\alpha} \sim 1$, roughly corresponding to doubled spatial periods of the forcing scale: $\mathrm{L}=2 L_{\mathrm{f}}, 4 L_{\mathrm{f}} \ldots$ At higher drives (e.g. at $1.2 \times 10^{3} \mathrm{~A} \mathrm{~m}^{-2}, R_{\alpha} \sim 3$ ) these multiple Lagrangian scales disappear, and the particle dispersion is determined by a single Lagrangian integral scale $\mathrm{L}_{\mathrm{L}}$. The emergence of this scale is correlated with the development of the hierarchy of turbulent scales in Eulerian wave number spectra.

The above results show a dramatic different manifestation of transition to turbulence in the Lagrangian and Eulerian frame. It is a process from multiple scales to single scale in the Lagrangian frame, while in the Eulerian frame, the transition to turbulence is manifested as the transition from a single scale to multiple scales.

\section{Lagrangian Scale}

In the Lagrangian frame of reference, we start with the most simple properties of the Lagrangian trajectories, the mean-square displacement (MSD) of the particle from its initial position $\left\langle\delta r^{2}\right\rangle$. The MSD of the particles is predicted to be ballistic at short time, and diffusive at longer time, based on the stochastic model ${ }^{12}$, as:

$$
\begin{aligned}
& \left\langle\delta r^{2}\right\rangle \approx\left\langle u^{2}\right\rangle t^{2}, \quad \text { at } t<<t_{L} \\
& \left\langle\delta r^{2}\right\rangle \approx 2\left\langle u^{2}\right\rangle T_{L} t, \quad \text { at } t>>t_{L}
\end{aligned}
$$




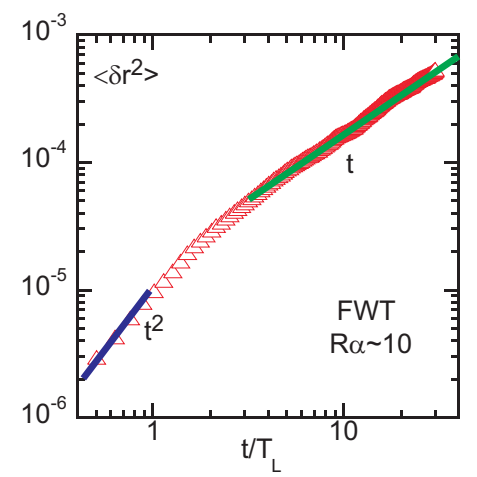

Fig. 3. Statistically averaged MSD $\left\langle\delta r^{2}\right\rangle$ versus time normalized by the integral time scale $t_{L}$, FWT at $60 \mathrm{~Hz} 2 \mathrm{~g}$ with a Reynolds number $R_{\alpha} \sim 10$.

where $t_{L}$ is the integral time, which can be obtained from the Lagrangian velocity autocorrelation function of the flow, as $t_{L}=\int_{0}^{\infty} \rho(t) d t$. The Lagrangian velocity autocorrelation function is $\rho(t)=\left\langle u\left[\vec{r}\left(t_{0}+t\right)\right] u\left[\vec{r}\left(t_{0}\right)\right]\right\rangle / \sigma^{2}$, where $\sigma^{2}$ is the velocity variance. The integral scale $t_{L}$ is indicative of the time scale when the particle lose its memory.

The statistically averaged MSD of particles in 2D turbulence has been reported to be diffusive confirming Taylor's diffusion law ${ }^{3,7}$. Recently it has been investigated in detail in a wide range of conditions ${ }^{6}$. The statistically averaged MSD versus time normalized by the integral time scale $t_{L}$ are shown for the one cases in Fig. 3 for $R_{\alpha} \sim 10$.

It can be seen from Fig. 3 that the behavior of the MSD for the flow at $R_{\alpha} \sim 4.4$ is as predicted in Eq. (2,3). For the short time regime, $t / t_{L}<1$, the particle dispersion is close to ballistic, showing a scaling of $\mathrm{t}^{2}$. For the long time limit, $t / t_{L}>>1$, the particle dispersion is diffusive, showing an linear trend. This is the case for all the MSD obtained in this experiment.

The single particle diffusion is illustrated in Fig. 4(a) for the low Reynolds number EMT experiments $\left(R_{\alpha} \sim 1-3\right)$. It shows that the merger of the dispersion curves plotted versus normalized time $\left(t / t_{L}\right)$ is observed only at higher drives, in the flows with developed turbulence spectra. The situation is different for the single particle diffusion curves at higher Reynolds number experiments (FWT, 60Hz, $R_{\alpha} \sim 3-10$ ), shown in Fig. 4 (b). The forcing scale for these experiments is the same since it is related to the Faraday frequency of $60 \mathrm{~Hz}$. The mean squared dispersions are plotted versus normalized time, $t / t_{L}$. All dispersion curves collapse onto one. This result suggests the existence of a universal spatial scale of particle dispersion related to the forcing scale as discussed in detail in Ref. 6. 

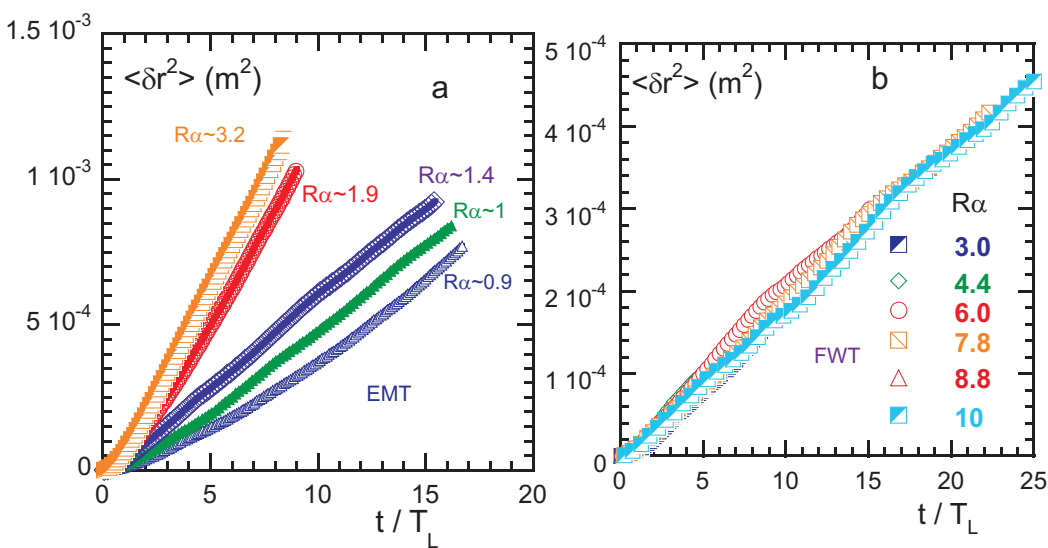

Fig. 4. MSD of EM turbulence and FW turbulence showing a transition from chaotic to turbulent flow. for (a): EMT turbulence and (b) FWT turbulence.

\section{Conclusions}

Lagrangian single particle statistics have been investigated experimentally in both chaotic and turbulent flows. The flows are generated through both the EMT and FWT. The energy density spectra of the flows show the transition to turbulence as a transition from a single energy injection scale to a hierarchy of scales in the energy inertial range. The Lagrangian energy spectra, however, show the transition to turbulence as the transition from multiple scales to a single scale dominant the dispersion.

The most studied parameter of the particle dispersion, the second order moment, or the mean-square displacement, shows a similar ballistic and diffusive law both the chaotic flow and turbulent flow. The MSD curves (plotted versus normalized $t, t / t_{L}$ ) for the experiments with the same forcing scale collapse onto a single curve at high drive for developed turbulence, indicating the convergence of the Lagrangian scale of particle dispersion to a single scale related to the forcing scale.

\section{Acknowledgments}

This work was supported by the Australian Research Council's Discovery Early Career Research Award (No. DE120100364).

\section{References}

1. N. Mordant, P. Metz, O. Michel and J.-F. Pinton, Phys. Rev. Lett. 87, 214501 (2001).

2. M. Bourgoin, N.T. Ouellette, H. Xu, J. Berg, and E. Bodenschatz. Science 311, 835(2006).

3. A. von Kameke, F. Huhn, G. Fernández-García, A.P. Muñuzuri, and V. Pérez-Muñuzuri, Phys. Rev. Lett. 107, 074502 (2011). 
H. Xia

4. N. Francois, H. Xia, H. Punzmann, and M. Shats, Phys. Rev. Lett. 110, 194501(2013).

5. H. Xia, M. Shats and G. Falkovich, Phys. Fluids 21, 125101 (2009).

6. H. Xia, N. Francois, H. Punzmann and M. Shats, Nature Communications 4 DOI: 10.1038/ncomms3013(2013).

7. M.K. Rivera and R.E. Ecke, http://arxiv.org/abs/0710.5888 (2007).

8. H. Xia, D. Byrne, G. Falkovich and M. Shats, Nature Physics 7, 321 (2011).

9. M. Shats, D. Byrne and H. Xia, Phys. Rev. Lett. 105, 264501(2010)

10. G. Boffetta, private communication.

11. H. Xia, H. Punzmann, G. Falkovich and M. Shats, Phys. Rev. Lett. 101, 194504 (2008).

12. G.I. Taylor, Proc. London Math. Soc. 20, 196 (1921).

13. M.G. Shats, H. Xia and H. Punzmann, Phys. Rev. E, 71, 046409 (2005) 